\title{
Molecular mechanisms of substrate-controlled ring dynamics and substepping in a nucleic acid-dependent hexameric motor
}

\author{
Nathan D. Thomsen ${ }^{a, b, 1}$, Michael R. Lawson ${ }^{a, b}$, Lea B. Witkowsky ${ }^{a, b}$, Song $\mathbf{Q u}^{\mathrm{a}, \mathrm{b}, 2}$, and James M. Berger ${ }^{\mathrm{a}, \mathrm{b}, 3}$

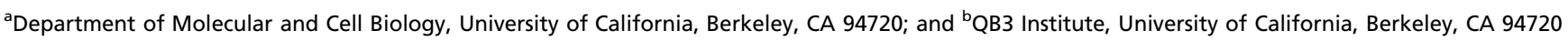 \\ Contributed by James M. Berger, October 17, 2016 (sent for review August 15, 2016; reviewed by Walter J. Chazin and Marcelo Nollmann)
}

\begin{abstract}
Ring-shaped hexameric helicases and translocases support essential DNA-, RNA-, and protein-dependent transactions in all cells and many viruses. How such systems coordinate ATPase activity between multiple subunits to power conformational changes that drive the engagement and movement of client substrates is a fundamental question. Using the Escherichia coli Rho transcription termination factor as a model system, we have used solution and crystallographic structural methods to delineate the range of conformational changes that accompany distinct substrate and nucleotide cofactor binding events. Small-angle X-ray scattering data show that Rho preferentially adopts an open-ring state in solution and that RNA and ATP are both required to cooperatively promote ring closure. Multiple closed-ring structures with different RNA substrates and nucleotide occupancies capture distinct catalytic intermediates accessed during translocation. Our data reveal how RNA-induced ring closure templates a sequential ATP-hydrolysis mechanism, provide a molecular rationale for how the Rho ATPase domains distinguishes between distinct RNA sequences, and establish structural snapshots of substepping events in a hexameric helicase/translocase.
\end{abstract}

ATPase | helicase | motor protein | transcription | translocase

$\mathbf{H}$ exameric helicases and translocases are motor proteins that play a central role in cellular transactions ranging from replication and repair to transcriptional regulation, chromosome packaging, and proteolytic homeostasis (1-4). Used to drive the processive and, at times, highly rapid movement of extended nucleic acid or protein chains through a central pore, ring-shaped motors face several challenges to their operation. One is that certain enzymes must transition through controlled ring opening and/or subunit assembly events to allow long, polymeric substrates that lack freely accessible ends to access interior motor elements (57). Another is that, once loaded, the molecular plasticity inherent to these assemblies must be harnessed to precisely coordinate ATP binding and hydrolysis between multiple subunits with the powering of substrate translocation, while at the same time alternating between tight and loose grips on the substrate to allow for processive movement. The substrate-dependent molecular rearrangements that underpin ring dynamics during these events remain poorly understood, not only for hexameric helicases and translocases, but for related ring-shaped switches as well.

The Escherichia coli Rho transcription termination factor is a well-established model system for understanding hexameric translocase and helicase function $(8,9)$. During termination, Rho uses a cytosine-specific RNA-binding domain appended to the $\mathrm{N}$ terminus of a RecA-type ATPase fold $(10,11)$ to bind nascent RNA transcripts at cytosine-rich sequences [known as Rho utilization (rut) sites] $(12,13)$. Once loaded, Rho consumes ATP to translocate $5^{\prime} \rightarrow 3^{\prime}$ toward a paused RNA polymerase, eventually promoting transcription bubble collapse and RNA release (14-19).

Structural studies have provided insights into the mechanics of RNA loading by Rho, imaging the helicase in both closed-circular and notched/lockwasher-shaped states (20-24). These findings have suggested that Rho monomers assemble into a pliant hexamer that can spontaneously open to allow nucleic acid entry into the translocation pore $(22,24,25)$, a property that has been observed in other hexameric helicases such as the eukaryotic MCM2-7 complex (26-28). By comparison, the structure of a closed-ring Rho complex bound to single-stranded RNA and the ATP-mimetic ADP. $\mathrm{BeF}_{3}$ has provided a picture of a prospective translocation intermediate of the motor (29). This work, combined with that of a prior landmark model for the human papillomavirus E1 helicase bound to single-stranded DNA and ADP (30) [as well as more recent work on the replicative helicase, DnaB (31)] has led to two general principles for understanding hexameric helicase function. One is that, as proposed first for the T7 gp4 protein (32), most hexameric helicases likely use a sequential, rotary ATPase mechanism similar to that used by the F1 ATPase to power translocation (33). Another is that both the relative order of hydrolysis between subunits and/or differences in subunit orientation within a motor can determine the polarity of nucleic acid translocation $(29,30,34,35)$.

Despite present-day insights, there remain many unanswered questions regarding the loading and translocation mechanism of Rho. For example, there has been no systematic assessment of the extent to which the various crystallographic states observed thus far correlate with the predominant structures adopted by

\section{Significance}

Hexameric, ring-shaped translocases are molecular motors that convert the chemical energy of ATP hydrolysis into the physical movement of protein and nucleic acid substrates. Structural studies of several distinct hexameric translocases have provided insights into how substrates are loaded and translocated; however, the range of structural changes required for coupling ATP turnover to a full cycle of substrate loading and translocation has not been visualized for any one system. Here, we combine low- and high-resolution structural studies of the Rho transcription termination factor, defining a set of conformational transitions that accompany substrate binding and translocations by a processive hexameric helicase.

Author contributions: N.D.T. and J.M.B. designed research; N.D.T., L.B.W., and S.Q. performed research; N.D.T., M.R.L., and J.M.B. analyzed data; and N.D.T., M.R.L., and J.M.B. wrote the paper.

Reviewers: W.J.C., Vanderbilt University; and M.N., Centre Nationale de la Recherche Scientifique.

The authors declare no conflict of interest.

Data deposition: The crystallographic data and structural models have been deposited in the Protein Data Bank, www.pdb.org [PDB ID codes 5J (RhoPolyU-P21), 5JJK (RhoPolyA), and 5JJL (RhoEmpty)].

'Present address: Gilead Sciences Inc., Foster City, CA 94404.

${ }^{2}$ Present address: Department of Molecular \& Experimental Medicine, Scripps Research Institute, La Jolla, CA 92037.

${ }^{3}$ To whom correspondence should be addressed at the present address: Department of Biophysics and Biophysical Chemistry, Johns Hopkins School of Medicine, Baltimore, MD 21205. Email: jmberger@jhmi.edu.

This article contains supporting information online at www.pnas.org/lookup/suppl/doi:10. 1073/pnas.1616745113/-/DCSupplemental. 
A

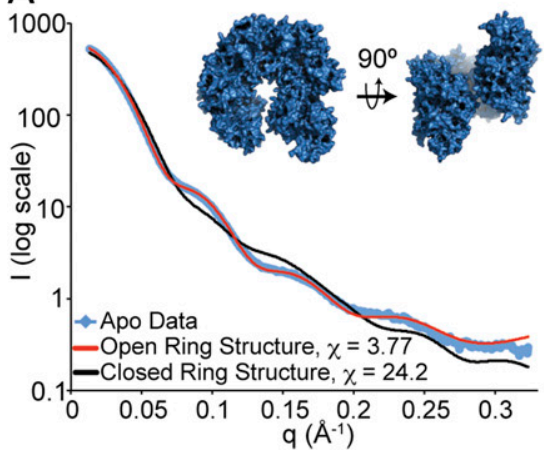

$B$

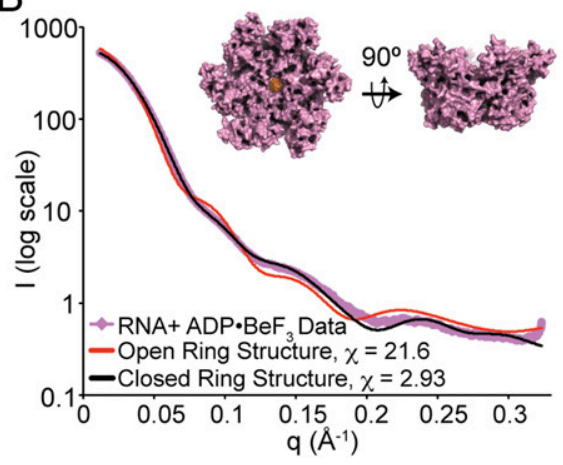

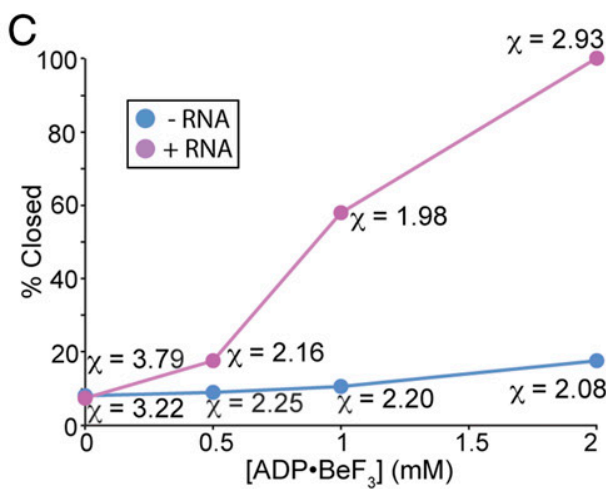

Fig. 1. SAXS analysis of apo and substrate-bound Rho complexes. (A) Scattering curve for apo Rho in solution (blue) closely matches the theoretical curve (red) calculated from a model based on the open-ring structure [PDB ID code 1PVO; Inset (22)], but not the theoretical curve (black) calculated from a closedring Rho model bound to RNA and $A D P \cdot B_{e} F_{3} .(B)$ Scattering curve for Rho in the presence of RNA (1:1 RNA:hexamer) and 2 mM ADP.BeF 3 (pink) closely matches the theoretical curve (black) calculated from the closed-ring Rho structure bound to RNA and ADP.BeF 3 determined here, but not the theoretical curve (red) calculated from an open-ring Rho model (PDB ID code 1PVO). (C) Plot of the percent-closed value obtained with FOXS vs. ADP.BeF 3 concentration shows that both RNA and ATP are required for Rho ring closure.

Rho in solution. The relative roles that ATP and RNA play in promoting ring opening vs. ring closing are similarly unresolved. At the same time, the range and types of movements that accompany ATP turnover events to support RNA translocation have yet to be fully defined. Likewise, it is unclear how the physical nature of the nucleic acid substrate itself-its chemical identity and base composition-feeds back to ATPase centers to influence motor activity. Answers to these questions are needed to understand fundamental aspects of hexameric helicase loading and translocation, not just by Rho, but by hexameric motor proteins in general.

To better define the physical basis by which substrate-coupled conformational changes underpin hexameric motor mechanism, we conducted both solution and crystallographic structural studies of $E$. coli Rho with the goal of characterizing novel conformational intermediates in conjunction with different RNA sequences and nucleotide-binding modes. Small-angle X-ray scattering (SAXS) confirmed that the current open- and closedring models imaged crystallographically correspond to dominant states adopted in solution in the absence and presence of substrates, respectively, and further establish that Rho ring closure occurs only when both RNA and ATP are present. Three crystal structures of Rho (including an experimentally phased structure at higher resolution than that determined previously) reveal ring rearrangements accompanying ATP cycling, which, together with comparisons to other oligomeric RecA-family motors, uncover a common allosteric linkage that physically connects nucleic-acid binding with ATPase activation in members of this widespread motor family. Together, our data highlight unexpected substeps in the Rho loading/translocation cycle and help explain previous research implicating RNA sequence as a regulator of Rho function in transcription termination (36-39).

\section{Results}

Both RNA and ATP Are Required to Drive Rho Ring Closure. To probe how Rho physically and differentially responds to the binding of substrates such as nucleotide and RNA, we first used SAXS to verify whether available crystal structures of the helicase correspond to major or minor population states in solution (Fig. $1 A$ and $B$ and SI Appendix, Fig. S1 $A$ and $B$ ) (40). SAXS is an ideal technique for analyzing the ensemble of states accessed in solution by macromolecular assemblies with conformational or configurational flexibility (41). SAXS data were initially collected on Rho in the absence of ligand and on Rho mixed with one molar equivalent per hexamer of a poly-pyrimidine RNA $\left(\mathrm{rU}_{12}\right)$ and $2 \mathrm{mM}$ ADP. $\mathrm{BeF}_{3}$, a nonhydrolyzable ATP analog.
Consistent with size-exclusion chromatography (SI Appendix, Fig. S1 $C$ and $D$ ), SAXS curves for Rho exhibited no sign of aggregation under any of the conditions tested, and distance distribution $\left(P_{(\mathrm{r})}\right)$ profiles, Guinier analysis, and Kratky plots also were consistent with a well-behaved, hexameric particle (SI Appendix, Fig. S2 and Table S1). Notably, data collected in the absence and presence of both RNA and $\mathrm{ADP} \cdot \mathrm{BeF}_{3}$ proved to be extremely close matches to the theoretical curves calculated from crystal structures of models of open- and closed-ring states of Rho, respectively (Fig. $1 A$ and $B$ ). Although our theoretical models do not fully account for interdomain and intersubunit flexibility [which would require polymer physics-based modeling of all flexible connections (42)], our data indicate that the openand closed-ring crystal structures seen previously for Rho are consistent with the conformations of native particles observed in solution.

To assess the individual contributions of RNA and nucleotide to ring closure, we next conducted SAXS experiments in either the presence or absence of RNA and over several ADP. BeF 3 concentrations. Data collected in the absence of RNA, but with $0,0.5,1$, or $2 \mathrm{mM} \mathrm{ADP} \cdot \mathrm{BeF}_{3}$, induced only modest changes in the scattering profile (SI Appendix, Fig. S1A). By contrast, scattering data collected in the presence of RNA exhibited distinct changes as $\mathrm{ADP} \cdot \mathrm{BeF}_{3}$ concentrations were increased ( $S I \mathrm{Ap}$ pendix, Fig. S1B). Data collected at intermediate concentrations of nucleotide could not be properly modeled by individual Rho crystal structures, so we used the programs FOXS and OLIGOMER to fit a two-component model consisting of both the open- and closed-ring Rho structures (SI Appendix, Tables S2 and S3) (43-45). The two-component analysis produced greatly improved fits for the intermediate states, indicating that, in the absence of RNA and nucleotide, the vast majority of Rho hexamers are open in solution and remain so even at high $(2 \mathrm{mM})$ $\mathrm{ADP} \cdot \mathrm{BeF}_{3}$ concentrations (Fig. $1 C$ ). By contrast, with the addition of RNA, nucleotide titration leads to ring closing in Rho, such that by $2 \mathrm{mM} A D P \cdot \mathrm{BeF}_{3}$, the hexamer population is dominated by the closed-ring state. Together, these data confirm that Rho forms an open lock-washer in solution and further demonstrate that both RNA and nucleotide are required to promote ring closure (5). This RNA- and ATP-dependent conformational change in Rho may correspond to the rate-limiting step previously observed in stopped-flow kinetics measurements of ATP hydrolysis (46), a concept supported by the marked acceleration of both ATPase and ring closure rates in response to primary site occupancy $(38,47)$. 
A
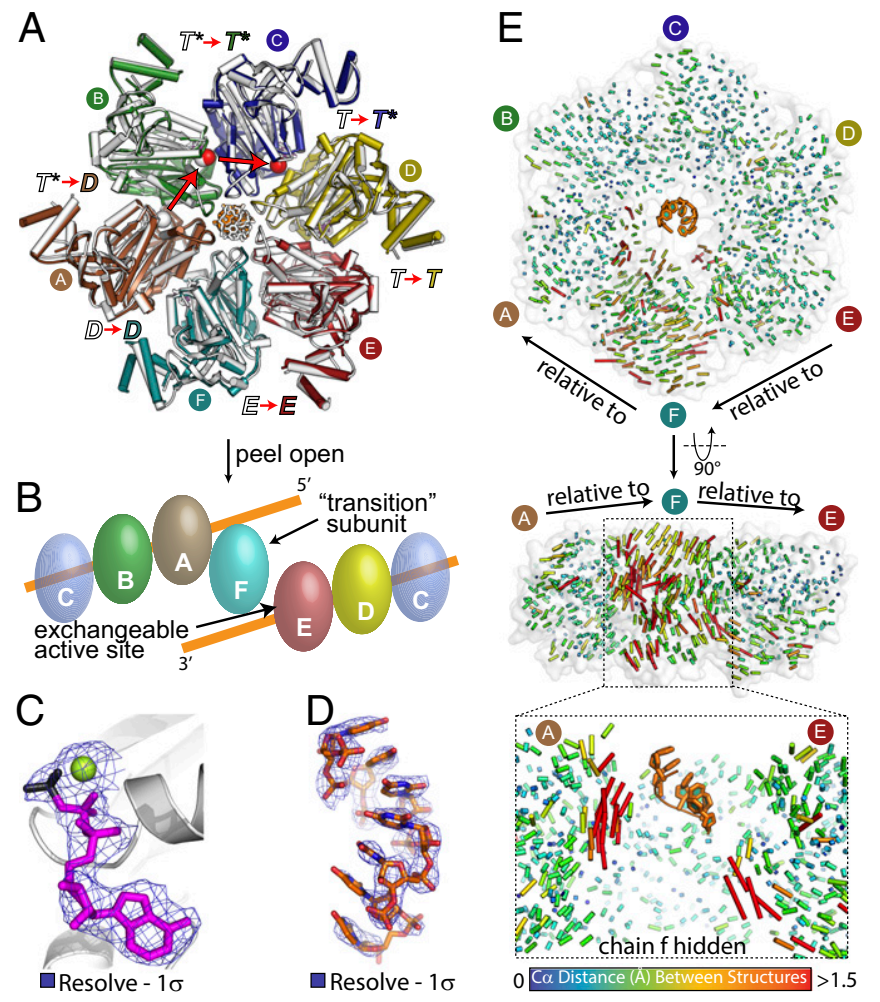

Fig. 2. Overall structure and ligand binding in a new Rho translocation in termediate. (A) Superposition of a new Rho.RNA.ADP.BeF structure (Rho $^{\mathrm{PolyU}^{-} \mathrm{P2}_{1}}$ colored and labeled by subunit), with the previously determined Rho.RNA.ADP.BeF ${ }_{3}$ structure (Rho ${ }^{\text {PolyU-P1 }}$, white). Presumptive ATP hydrolysis states ( $T$, ATP bound; $T^{*}$, ATP hydrolysis; $D$, product; $E$, exchange) are labeled in color (Rho ${ }^{\text {PolyU-P2 }_{1}}$ ) or white (Rho ${ }^{\text {PolyU-P1 }}$ ). (B) Schematic of closed-ring Rho hexamer (flattened on page) bound to RNA (one complete turn) highlighting the righthanded helical rise of the $A / B / C / D / E$ interfaces that are bound to RNA, and the position of subunit $F$ in transition between the top and bottom steps of the RNA-binding staircase. Subunit $C$ is shown twice to connect the two sides. (C) Experimental (density modified, SAD phased, Rho ${ }^{\text {PolyU-P2 }}{ }_{1}$ ) electron density around one of the bound nucleotides (ADP, magenta; $\mathrm{BeF}_{3}$, black; $\mathrm{Mg}^{2+}$, yellowgreen). (D) Experimental (density modified, SAD phased, Rho ${ }^{\text {PolyU-P2 }_{1}}$ ) electron density around the bound RNA (orange). (E) Intersubunit distance vectors calculated independently for each intersubunit interface between the Rho ${ }^{\text {PolyU-P1 }}$ and Rho ${ }^{\text {PolyU-P2 }_{1}}$ structures. Vectors were calculated relative to either the clockwise-adjacent subunit (Upper) or to the counterclockwise-adjacent subunit (Lower) revealing that the new Rho ${ }^{\text {PolyU }^{-P 2_{1}}}$ structures differ from the original Rho ${ }^{\text {PolyU-P1 }}$ model primarily in the position of subunit $F$ and in the $Q$ and $R$ loops of subunits $A$ and $E$.

\section{A Closed-Ring Rho State Manifests a Sequential Shift in ATPase} Status. Having established that existing crystallographic structures are suitable models for the open and closed Rho states in solution, we next set out to determine whether different substratebound, closed-ring forms of Rho might exist. We postulated that different RNA sequences or nucleotide-binding conditions might influence the formation of such intermediates. Because our initial closed-ring Rho crystals were difficult to work with (hereafter referred to as Rho ${ }^{\text {PolyU-P1 }}$, in reference to the RNA sequence and space group in which the protein crystallized) (48), we set out to find a new crystal packing arrangement that could be obtained in the presence of different RNA and nucleotide conditions, but that still possessed at least one complete Rho hexamer per asymmetric unit (so as to image the particle in the absence of crystal symmetry influences). We therefore conducted a new, broader set of screens with purified Rho in the presence of RNA and nucleotide, discovering a crystal form belonging to the space group $\mathrm{P} 2_{1}$ that satisfied these criteria (Methods).
From the new Rho form, we were able to experimentally phase and refine a higher-resolution (2.6 ̊) structure of an intact hexamer bound to seven nucleotides of a centrally associated poly-U RNA, and that displayed electron density for $\mathrm{ADP} \cdot \mathrm{BeF}_{3} \cdot \mathrm{Mg}^{2+}$ bound to all six ATP-binding sites (hereafter referred to as Rho ${ }^{\text {PolyU-P2 }_{1}}$ ) (Fig. $2 A-D$ and Table 1 ). The experimental phases and higher resolution allowed us to correct two small building errors in previous Rho structures (SI Appendix, Fig. S3). A global alignment of the new Rho ${ }^{\text {olyU-P2 }_{1}}$ model with the original Rho ${ }^{\text {PolyU-P1 }}$ state revealed that the two hexamers are highly similar structurally (Fig. $2 A$ ), with a root mean squared deviation (rmsd) for all shared backbone $\mathrm{C} \alpha$ atoms of $1.55 \AA$. As seen previously, five subunits of the Rho ${ }^{\text {PolyU-P2 }_{1}}$ state form a smoothly ascending spiral staircase that wraps around a single-stranded RNA helix; the sixth (subunit F) occupies a "transit" position midway between the uppermost (A) and bottommost (E) protomers to close the ring (Fig. $2 B$ ). In addition, four classes of nucleotide-binding sites were again evident at the six intersubunit interfaces. Using criteria such as nucleotide B factors [a measure of positional accuracy, or "orderedness" (49)] and coordination geometry, these sites have been classified as corresponding to either "exchange" $(E)$, "ATP-bound" $(T)$, "hydrolysis" $\left(T^{*}\right)$, or product $(D)$ states along the ATPase cycle (Fig. 2A) (29).

Globally, the conformations of the ATPase sites and the sequential order of ATPase states in the new Rho ${ }^{\mathrm{PolyU}_{-} \mathrm{P}_{1}}$ Rho model initially appeared similar to those seen in the original Rho ${ }^{\text {PolyU-P1 }}$ structure, with the modeled nucleotides refining to a relative Bfactor distribution consistent with the formation of three tight and three weak ATP-binding environments as observed biochemically (50-55) (SI Appendix, Fig. S4). However, inspection of the distance vectors calculated between $\mathrm{C} \alpha$ atoms for the Rho ${ }^{\text {PolyU-P1 and }}$ Rho ${ }^{\text {PolyU-P2 }_{1}}$ models revealed that subunit F, as well as the RNAbinding $\mathrm{R}$ loops of subunits $\mathrm{E}$ and $\mathrm{A}$, have moved with respect to one other around a vector approximately parallel to the ring axis (Fig. 2E).

Because the A, F, and E subunits are thought to represent the likely sites for the release of nucleotide hydrolysis products and the binding of fresh ATP within the hexamer (29), we elected to compare the nucleotide-binding regions of the two Rho models in more detail. This analysis uncovered pronounced changes in some of the nucleotide-binding sites, which, in turn, revealed a shift in the pattern of functional states between the Rho ${ }^{\text {PolyU-P1 }}$ and Rho ${ }^{\text {PolyU-P2 }}$ forms of Rho (Figs. $2 A$ and 3 ). The most striking change was that the presumptive catalytic water molecules seen in subunit A and B active sites of the initial closed-ring structure now appear in the catalytic centers of subunits $\mathrm{B}$ and $\mathrm{C}$ in the new structure (Figs. $2 A$ and $3 A-C$ and SI Appendix, Fig. S5 $A-C$ ). Moreover, each of these waters also has shifted its local position from a nonideal location in the Rho ${ }^{\text {PolyU-P1 }}$ model to take up a direct, in-line attack configuration with the $\mathrm{BeF}_{3}$ group of the bound nucleotide in the Rho ${ }^{\text {PolyU-P2 }_{1}}$ structure (Fig. $3 B$ ). Repositioning of the waters is mediated at both interfaces by a conserved arginine [the "arginineswitch" (RS), R269], the catalytic glutamate (CE) residue (E211) that polarizes the water to assist hydrolysis, and a backbone carbonyl (G337) of the adjacent protomer. The glycine-mediated contact was not observed in the prior Rho ${ }^{\text {PolyU-P1 }}$ structure. Consistent with biochemical studies implicating their importance to overall Rho function $(10,56-60)$, a conserved network of ion pairs at the $\mathrm{B} / \mathrm{C}$ and C/D interfaces-which runs from the RNA-binding pore to the ATPase center-appears to help orient amino acids responsible for in-line placement of the attacking water (SI Appendix, Fig. S6). Overall, the new placement for the catalytic waters in

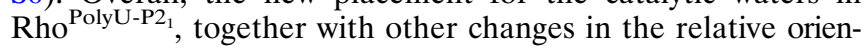
tations of catalytically important amino acids (Fig. 3 and SI Appendix, SI Discussion), supports the proposed existence of hydrolysis-competent active sites in the closed-ring Rho states (29). This shift also advances the relative position of the two $T^{*}$ states by one subunit 
Table 1. Data collection, phasing and refinement

\begin{tabular}{|c|c|c|c|c|}
\hline Parameter & Rho ${ }^{\text {PolyU-P2 }_{1}}$ & Rho ${ }^{\text {PolyU-P2 }}$ & Rho PolyA & Rho Empty \\
\hline \multicolumn{5}{|l|}{ Data collection } \\
\hline Space group & $\mathrm{P} 2_{1}$ & $\mathrm{P} 2_{1}$ & $\mathrm{P} 2_{1}$ & $\mathrm{P} 2_{1}$ \\
\hline \multicolumn{5}{|l|}{ Cell dimensions } \\
\hline$a, b, c, \AA$ & $68.72,198.8,111.8$ & $69.00,198.6,111.4$ & $68.76,199.2,112.2$ & $69.09,199.1,111.7$ \\
\hline \multirow[t]{2}{*}{$\alpha, \beta, \gamma,{ }^{\circ}$} & $90.0,104.8,90.0$ & $90.0,104.4,90.0$ & $90.0,104.8,90.0$ & $90.0,105.0,90.0$ \\
\hline & Peak & Remote & remote & remote \\
\hline Wavelength & 0.9796 & 1.116 & 1.116 & 1.116 \\
\hline Resolution, Å & $50-2.95(3.06-2.95)$ & $50-2.60(2.69-2.60)$ & $50-3.15(3.26-3.15)$ & $50-3.2(3.31-3.20)$ \\
\hline$R_{\text {sym }}$ or $R_{\text {merge }}$ & $9.1(46)$ & $8.1(63)$ & $12.1(60)$ & $12.8(58)$ \\
\hline$I / \sigma l$ & $10.8(2.4)$ & $14(1.8)$ & $9.4(2.0)$ & $8.1(1.8)$ \\
\hline Completeness, \% & $98.2(97.1)$ & $99.7(99.1)$ & $99.0(99.5)$ & $96.2(96.4)$ \\
\hline Redundancy & $5.6(5.2)$ & $3.1(3.1)$ & $2.9(2.9)$ & $2.8(2.8)$ \\
\hline \multicolumn{5}{|l|}{ Refinement } \\
\hline Resolution, Å & & $47.4-2.6$ & $49.8-3.15$ & $49.5-3.2$ \\
\hline No. of reflections & & 88,380 & 49,848 & 46,060 \\
\hline$R_{\text {work }} / R_{\text {free }}$ & & $21.8 / 24.6$ & $23.3 / 26.5$ & $23.2 / 26.6$ \\
\hline \multicolumn{5}{|l|}{ No. of atoms } \\
\hline Protein & & 19,164 & 18,696 & 18,888 \\
\hline Ligand/ion & & 341 & 347 & 325 \\
\hline Water & & 271 & 19 & $15^{\dagger}$ \\
\hline \multicolumn{5}{|l|}{ B factors } \\
\hline Protein & & 67.9 & 80.7 & 73.7 \\
\hline Ligand/ion & & 53.6 & 69.9 & 66.6 \\
\hline Water & & 45.2 & 64.5 & 46.6 \\
\hline \multicolumn{5}{|l|}{ rmsds } \\
\hline Bond lengths, Å & & 0.006 & 0.006 & 0.006 \\
\hline Bond angles, $^{\circ}$ & & 0.575 & 0.560 & 0.691 \\
\hline
\end{tabular}

Values in parenthesis correspond to the highest resolution bin.

${ }^{\dagger}$ Only waters involved in octahedral coordination of the active site $\mathrm{Mg}^{2+}$ ions were modeled.

toward the lone $E$ state and adds a second $D$ state compared with the Rho ${ }^{\text {PolyU-P1 }}$ structure determined previously (Fig. $2 A$ ).

Intersubunit Relationships in the Nucleotide-Exchange Region Mirror Those of an Open-Ring State. A key supposition of the rotary cycling model based on the original Rho ${ }^{\text {Polyu-P1 }}$ crystal form has been that the $\mathrm{E} / \mathrm{F}$ interface, which is relatively open and solvent exposed, corresponds to a nucleotide-exchangeable $(E)$ site where substrate can bind to or product can dissociate from the hexamer, respectively (29). To test this idea, we back-soaked nucleotide out of the new Rho ${ }^{\mathrm{PolyU}^{-} \mathrm{P2}{ }_{1}}$ crystal form and determined the structure of the helicase by X-ray crystallography. Inspection of all active sites in the model revealed very weak and discontinuous electron density for the nucleotide-binding site of subunit $\mathrm{E}$, indicating a very low occupancy for $\mathrm{ADP} \cdot \mathrm{BeF}_{3}$ that precluded modeling of nucleotide into the site (nucleotide density in the other active sites was unchanged) (Fig. $4 A$ and $B$ ). Intersubunit distance vectors calculated between the back-soaked structure (referred to hereafter as Rho ${ }^{\text {Empty }}$ ) and the fully occupied Rho ${ }^{\text {PolyU-P2 }_{1}}$ structure show that the relative positions of subunits $\mathrm{E}$ and $\mathrm{F}$ shift by $\sim 1.7 \AA$ with respect to one another, but that the rest of the subunits in the hexamer undergo only minor positional changes (Fig. 4C). A comparison of the $\mathrm{E} / \mathrm{F}$ interface in the presence and absence of $\mathrm{ADP} \cdot \mathrm{BeF}_{3}$ reveals an increased separation of the two subunits that moves the Arg finger out of the active site and that buries $\sim 120 \AA^{2}$ less total surface area at the interface (Fig. $4 D$ ). Together, these observations further confirm the proposition that the $\mathrm{E} / \mathrm{F}$ active site corresponds to a nucleotide-exchange point, and show that product release coincides with an increased opening of the subunit interface at this catalytic center. Interestingly, upon the disappearance of nucleotide from the E state, the relative $\mathrm{B}$ factors for the nucleotide in the active site of subunit $\mathrm{F}$ also increase relative to other Rho structures (SI Appendix,
Fig. S4), suggesting that product release may cooperatively weaken the association of nucleotide in the immediately adjacent $D$ state.

Inspection of the $\mathrm{E} / \mathrm{F}$ active site shows that the $E$ state is unique within the closed-ring Rho hexamer in adopting a relative intersubunit orientation markedly different from that of the other protomers. This altered configuration arises due to a transition of subunit $F$ (which is flanked by subunits $A$ and $E$, respectively) from the top step of the helical RNA staircase to the bottom (Fig. $2 B$ ). Subunit $\mathrm{F}$ is also the only protomer that does not contact RNA in any closed-ring structure determined so far, and its interface with subunit $\mathrm{E}$ in the Rho ${ }^{\text {Empty }}$ structure represents a completely substrate-free component of the catalytic cycle. Given these considerations, we compared the intersubunit positions of the $\mathrm{E} / \mathrm{F}$ protomer conformation of the back-soaked model with those of a completely RNA-free, open-ring Rho structure of Rho determined previously. Interestingly, the intersubunit orientation of motor domains in the open-ring structure [Protein Data Bank (PDB) ID code 1PVO], which all assemble with a left-handed helical pitch, proved to be a very close match to the orientation of the E/F protomer pair in both the Rho ${ }^{\text {Empty }}$ and Rho ${ }^{\text {PolyU-P2 }_{1}}$ structures, but distinct from other subunit dimers in the RNA-bound models (which adopt a right-handed pitch) (Fig. 4E). This congruency indicates that, upon ATPase product and RNA release, a single interface in the Rho hexamer relaxes to an energetically favorable conformation present in the open-ring Rho structure. Therefore, the nucleotide-dependent structural transition, which promotes ring opening and closing during RNA loading, also plays a direct role in catalytic cycling.

Sequence and ATPase State-Specific Differences in RNA Binding by Rho. The ATPase activity of E. coli Rho has been well-established to depend not just on RNA binding (61), but also on RNA sequence, 


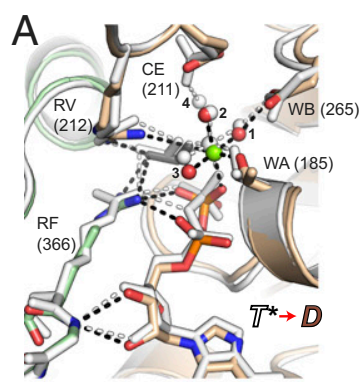

Key:

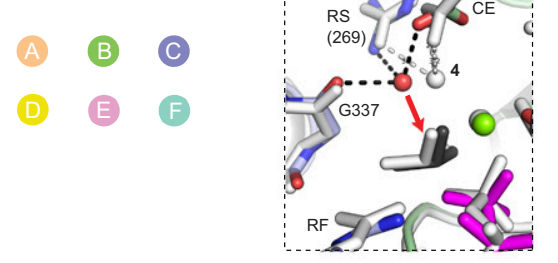

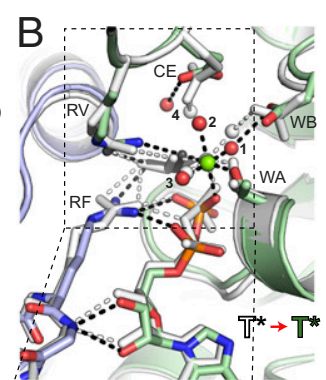
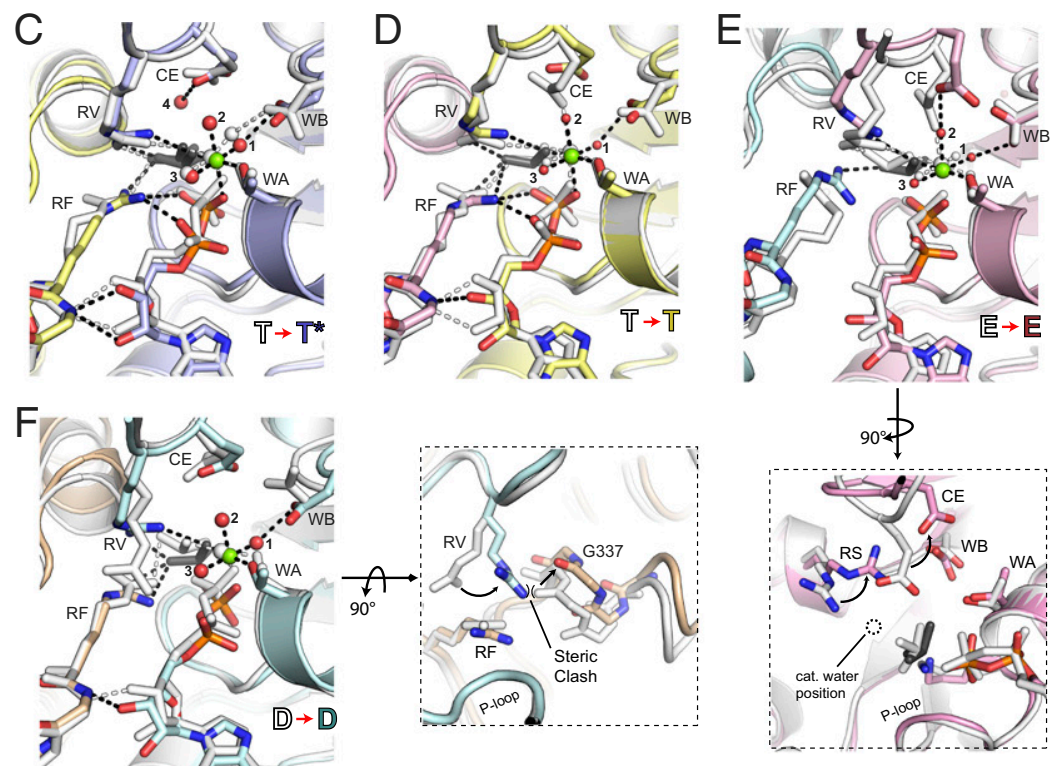

Fig. 3. Changes in ADP.BeF $F_{3}$ coordination as revealed by superpositions between six Rho ${ }^{\text {PolyU-P1 }}$ (white) and Rho ${ }^{\text {Polyu-P2 }}{ }_{1}$ (colored) ATPase centers. In all subunits, changes are consistent with a partial shift in catalytic status of the six active sites around the ring. RF, arginine finger; RS, arginine switch; RV, arginine valve; WA, Walker A; WB, Walker B. (A) Subunit A/B interface. While a water molecule is observed to associate with the catalytic glutamate (CE) at this site in Rho ${ }^{\text {Polyu-P1 }}$ (water 4), it is not observed in the new Rho ${ }^{\text {Polyu-P2 }_{1}}$ structure. (B) Subunit B/C interface. Both catalytic centers show bonding interactions (dashed lines) characteristic of the $T$ and $T^{*}$ states. $B$, Inset shows a zoomed-in view of the catalytic center with the RV removed (for clarity); whereas the prospective attacking water molecule in

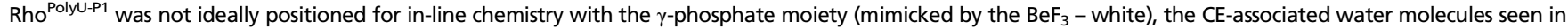
the $T^{*}$ states of the new Rho ${ }^{\text {Poly U-P2 }_{1}}$ are positioned for ideal in-line attack (red arrow). (C) Subunit C/D interface. Concomitant with the disappearance of the catalytic water molecule in the $A / B$ subunit interface of the Rho ${ }^{\mathrm{POlyU}^{-P 2}{ }_{1}}$ structure, a catalytic water molecule appears in the C/D subunit interface compared with the Rho ${ }^{\mathrm{Poly}_{\mathrm{O}}-\mathrm{P} 1}$ structure. $(D)$ Subunit D/E interface. This site retains contacts characteristic of the $T$ state in both structures. The CE in Rho ${ }^{\text {PolyU-P2 }_{1}}$ has broken its atypical contact with the $\mathrm{Mg}^{2+}$ ion observed in Rho PolyU-P1 suggesting that it is transitioning to a slightly more active arrangement. $(E)$ Subunit E/F interface. The arginine finger in the Rho ${ }^{\text {PolyU-P1 }} E$ state appears to be pointing out of the active site, whereas the arginine finger in Rho ${ }^{\text {PolyU-P2 }}{ }_{1}$ clearly points into the active site and coordinates the bound $\mathrm{BeF}_{3}$. However, the arginine valve in the Rho ${ }^{\mathrm{Poly}_{\mathrm{U}} \mathrm{P2}{ }_{1}}$ structure is pulled slightly out of the active site, resulting in the same number of contacts between the arginine groups and the $\mathrm{BeF}_{3}$ molecule. The catalytic glutamate in this active site is in an atypical conformation in both structures, interacting with the $\mathrm{Mg}^{2+}$ ion directly

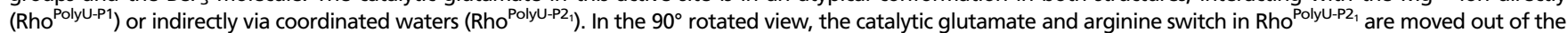

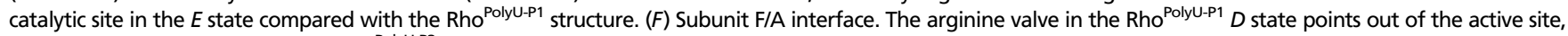
whereas the arginine valve in the Rho ${ }^{\text {Polyu-P2 }_{1}}$ structure points into the active site and coordinates the bound BeF $F_{3}$. In the $90^{\circ}$ rotated view, the arginine valve now points into the active site due to an opening of the intersubunit interface in Rho ${ }^{\text {PolyU}^{-P 2}{ }_{1}}$ relative to Rho ${ }^{\text {PolyU-P1 }}$ that relieves a steric clash between the guanidinium group of the arginine and G337 of the adjacent subunit.

with pyrimidine-rich RNAs providing a greater increase in ATP turnover compared with purine-rich substrates $(37,38)$. To better understand this dependency, we crystallized and determined the structure of Rho in complex with a poly-purine RNA $\left(\mathrm{rA}_{12}\right)$ and $\mathrm{ADP} \cdot \mathrm{BeF}_{3}$ (hereafter referred to as Rho ${ }^{\text {PolyA }}$ ) and compared it to the other Rho structures determined thus far (Fig. 5). Notably, the Rho-poly-A complex cocrystallized in the same P2 space group and unit cell as the new closed-ring Rho model bound to poly-U. The poly-A substrate similarly forms an overwound, single-stranded RNA helix in the Rho pore that is highly similar to that formed by poly-U (Fig. 5 and SI Appendix, Fig. S7), despite the extra bulk afforded by the additional fivemember ring on the purine bases. This finding indicates that the tight coiling of RNA seen in the original Rho model is not an effect of RNA sequence or crystal packing, but likely reflects the standard manner by which the helicase encircles substrate during translocation.

Close inspection of the poly-A Rho model with its poly-U counterparts reveals notable differences in the specifics of RNA binding (Fig. 5 and SI Appendix, SI Discussion). Interestingly, these differences are evident not just between purine- and pyrimidine-bound Rho hexamers, but also between the different poly-U models imaged thus far (Fig. $5 A-C$ ). In particular, the $\mathrm{Rho}^{\mathrm{PolyU}^{-} \mathrm{P}_{2}}$ and Rho ${ }^{\mathrm{PolyA}}$ structures lack electron density for the $5^{\prime}$-most base present in the original Rho ${ }^{\text {PolyU-P1 }}$ structure, and instead manifest new density for two additional bases at the $3^{\prime}$ end of the RNA (Fig. 5 and SI Appendix, Fig. S7 B-G). However, the bound RNAs also show conformational differences between these three structures, with the additional $3^{\prime}$ nucleotides visible in the poly-A-bound Rho model extending in a straight line from the helicase pore, but in the new poly-U structures folding back into a tight kink that deviates from the interior RNA spiral configuration but maintains base-stacking interactions (Fig. 5 and SI Appendix, Fig. S7). The U-U kink seen in the poly-U models allows for a network of contacts to be formed between the ribose $2^{\prime}-\mathrm{OH}$ and the uracil $\mathrm{O} 2$ atom of the penultimate nucleotide, a newly evident water molecule, and Lys-326 of both subunits B and C (the latter of which alters a contact that had been seen previously between this invariant amino acid and the backbone phosphate of RNA in the original Rho ${ }^{\mathrm{Poly} U-\mathrm{P} 1}$ hexamer) (Fig. 5 A-C and SI Appendix, Fig. S8). Interestingly, in analyzing the Rho ${ }^{\text {Empty }}$ structure, we observed electron density for the additional, kinked $3^{\prime}$ bases of RNA similar to that present in Rho ${ }^{\mathrm{PolyU}^{-}-\mathrm{P2}_{1}}$; however, this model also exhibited density for the $5^{\prime}$-most base seen in Rho ${ }^{\text {PolyU-P1 }}$ (Fig. $5 B$ and SI Appendix, Fig. S7 B-D). Thus, upon loss of nucleotide and opening of the $E$-state active site, the polyU RNA bound to Rho ${ }^{\text {Empty }}$ appears to adopt a hybrid conformation between the original Rho ${ }^{\text {PolyU-P1 }}$ crystal form and the new Rho ${ }^{\text {PolyU-P2 }_{1}}$ crystal form.

A Conserved Allosteric Helix Coordinates Cross-Talk Between Subunits and Their Catalytic Centers. In considering how changes in the ATPase centers of our closed-ring Rho models might be coupled to RNA binding, we used the higher-resolution structures 

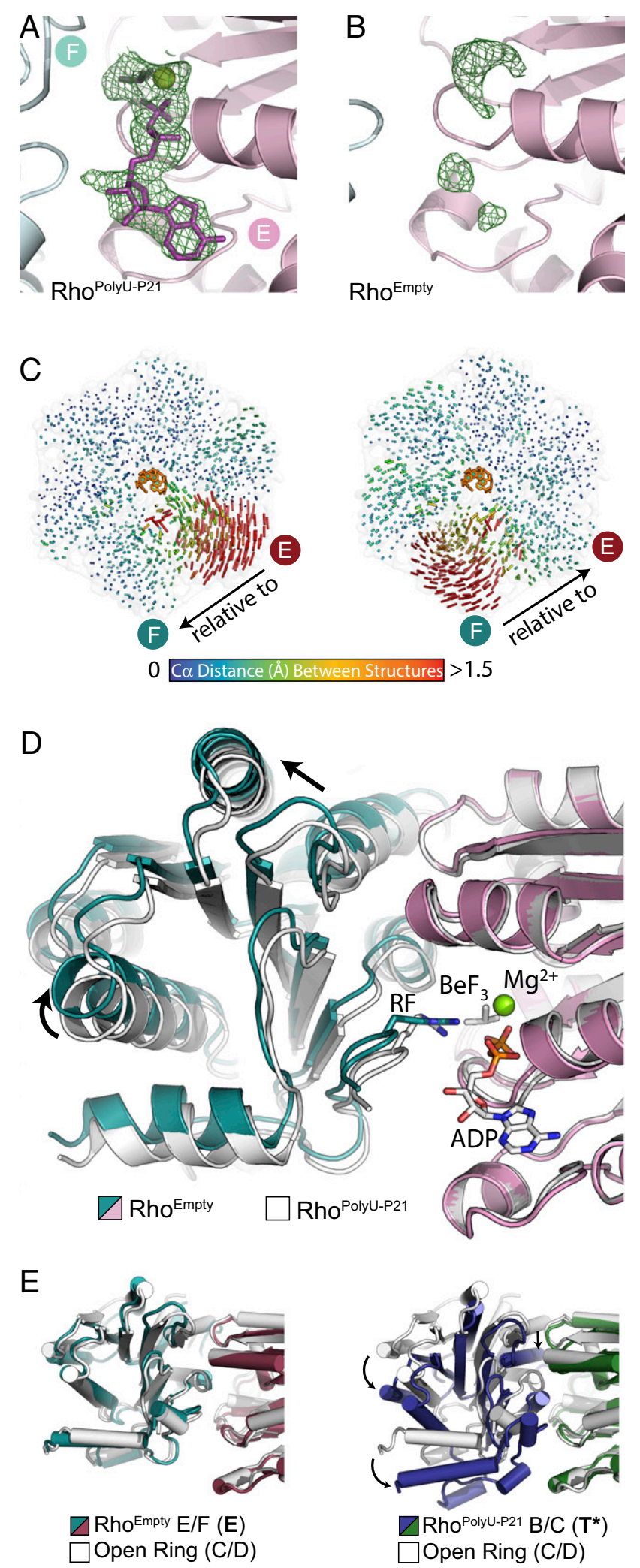

Fig. 4. Opening of the active site in the exchange $(E)$ state (subunit $E$ ) accompanies nucleotide release. $(A)$ Simulated annealing omit map of the $E$-state in Rho ${ }^{\mathrm{PolyU}_{-} \mathrm{P2}_{1}}$ reveals strong density for the bound nucleotide. (B) Simulated annealing omit map of the $E$ state in the Rho ${ }^{\text {Empty }}$ structure reveals poor density, suggesting low nucleotide occupancy and/or order. (C) Intersubunit distance vectors calculated independently for each intersubunit interface and comparing Rho ${ }^{\text {Empty }}$ and Rho ${ }^{\text {PolyU-P2 }_{1}}$ structures. The vectors reveal that the only significant intersubunit changes between these determined here to look more closely at the linkages that mediate intersubunit contacts between the nucleic acid and nucleotide binding sites. By coaligning all six pairwise interfaces of the Rho ${ }^{\text {PolyU-P2 }_{1}}$ model, using the ATP-binding P-loop as an reference point, we found that a single $\alpha$-helix $(R \alpha 1)$, which contains residues responsible for forming the salt-bridge network linking the RNA binding pore to the ATPase center, undergoes a large range of previously unnoticed hinge movements (Fig. $6 A$ and SI Appendix, Fig. S6). The $\mathrm{N}$ terminus of this helix (which corresponds to the "R-loop" of Rho and the "L2"-loop of RecA helicases such as DnaB and T7 gp4) binds RNA via K326 (Fig. 5 ), whereas the $\mathrm{C}$ terminus of this helix contains backbone carbonyls that interact directly with the catalytic water molecule and the arginine-based $\gamma$-phosphate sensors in the two $\mathrm{T}^{*}$ states (Figs. $3 B$ and $6 B$ ). This movement appears to be caused by an intersubunit levering motion of $\sim 10 \AA$ at the helix's $\mathrm{N}$ terminus, which alters the interactions in an ordered manner that correlates with the ATPase state of each subunit (Fig. $6 B$ and Movie S1). This observation suggests that R-loop-mediated RNA binding, aided by interactions within an intersubunit allosteric network, not only positions the catalytic glutamate via the arginine switch residue as described (29), but also directly controls the position of the arginine finger, arginine valve, and catalytic water by coupled movements with the associated $\mathrm{R} \alpha 1$ helix.

In conducting this analysis, we note that the $\alpha$-helix corresponding to $\mathrm{R} \alpha 1$ is a conserved feature of the RecA fold and is

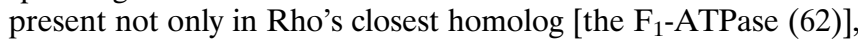
but also in more distantly related RecA-family hexameric helicases and translocases. In all cases, the C-terminal carbonyls of the helix equivalent to $\mathrm{R} \alpha 1$ contact amino acids that interact with the $\gamma$-phosphate moiety (and catalytic waters when present) and that appear poised to help control the active-site organization of their respective hexameric motors through a similar mechanism (Fig. $6 C-E)(31,62,63)$. These comparisons indicate that this helix, which lies downstream of the R/L2 loop region, is likely involved in allosterically coupling substrate movements to ATPase status in RecA-like motors in general.

\section{Discussion}

Possible Role for Ring Distortion and Strain in the Mechanism of Rho and Other Hexameric Motors. In the present study, we have used solution-based structural methods and X-ray crystallography to better understand how a RecA-family hexameric motor protein, Rho, couples changes in nucleotide status to conformational transitions that underpin ring loading and translocation events. Our SAXS data confirm that distinct open- and closed-ring structures are accessed before and after RNA engagement, respectively (Fig. $1 A-C$ ), suggesting that the transition between these two states likely occurs as a relatively simple rigid body rotation of subunits with respect to each other (Movie S2). Notably, our studies indicate that at nucleotide concentrations approaching those in the cell [values between 2 and $10 \mathrm{mM}$ ATP have been measured for E. coli (64-66]], Rho spontaneously and preferentially occupies an open-ring state, a form that is well suited for engaging nascent RNA transcripts. A need for contacts engendered by both substrate nucleic acid and ATP binding to cooperatively induce ring closure is consistent with the idea that formation of a translocation-competent state may capture and

structures are at the E/F interface. $(D)$ Superposition of chain $E$ from the

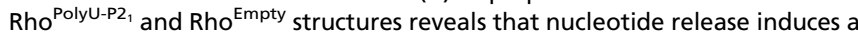
rigid body shift in chain $\mathrm{F}$ (arrows) that causes the active site to open. The arginine finger (RF), which also changes position, is labeled. (E) Structural alignments showing that the E/F subunit ( $E$ state) interface of Rho ${ }^{\text {Empty }}$ aligns almost perfectly with an open-ring intersubunit interface (Left), as opposed to the B/C subunit ( $T^{*}$ state) subunit interface of Rho ${ }^{\text {PolyU-P2 }_{1}}$, which has adopted a right-handed orientation (Right; black arrows). 

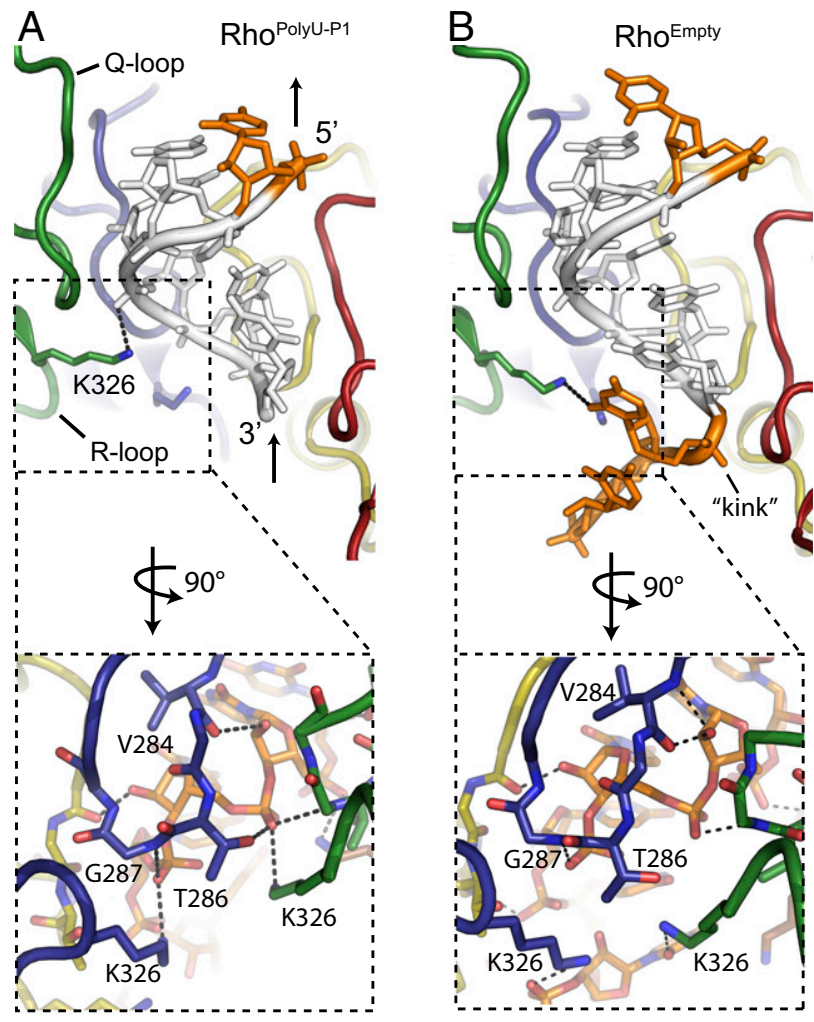

harness strain as a motive force to help promote nucleotide cycling. Consistent with this model, the formation of a relaxed, left-handed intersubunit orientation characteristic of the open-ring structure is seen only in the product release state, which also releases its grip on RNA to reset the RNA binding cycle (Fig. $4 E$ ).

Strain imparted by substrate-induced conformational changes may underlie ATP cycling in other, more distantly related hexameric helicases. For example, recent structures of DnaB-family helicases have shown that the ATPase domains of these hexameric motors adopt an uncoupled, planar conformation in the absence of nucleic acid substrate (67), but form a steep, right-handed helical conformation with a single open interface when DNA and GDP.AlF 4 are present $(31,67)$. Electron microscopy data on the eukaryotic minichromosome maintenance proteins (MCMs) have revealed that a left-handed open-ring conformation is the default state of metazoan MCM hexamers (27), and that the Cdc45.GINS complex, along with ATP and DNA, are needed to drive the formation of a stable closed ring with an internal, right-handed pitch $(26,34)$. Together, these studies support the idea that nucleic acids cooperatively activate ATP hydrolysis in many ringshaped RNA and DNA translocases by serving as a template for the asymmetric assembly of ATPase subunits. This asymmetry, in turn, promotes the formation of a defined sequence and order of active-site states $(29,30)$, which allows the energetics of cooperative substrate binding in one subunit to be allosterically coupled to cooperative product release in another. One exception to this trend may be the papilloma virus E1 helicase, in which five of its six subunits have been seen to adopt a right-handed spiral as part of closed-ring hexamer in the absence of ATP and DNA (68).

RNA Sequence Dependencies of the Rho Functional Cycle. In addition to the insights afforded into ring dynamics and the establishment of a sequential ATPase order, the structures presented here help to explain some of the known effects that RNA sequence has on Rho's ATPase activity. In general, the molecular basis for substrate-specific sequence dependencies in hexameric helicases and translocases has remained relatively ill-defined. Some ring-shaped helicases, such as T7 gp4, decelerate when confronted with GC-rich duplexes (69), indicating that motor function can be impacted by substrate stability. For Rho, transcription termination activity is stimulated by cytosine-rich sequences (13), due in part to the recognition requirements of its primary RNA (rut)-binding sites $(10,38)$ and also to the ability of primary site occupancy to promote Rho ring closure as demonstrated in the accompanying study (47). However, Rho's RNA-stimulated ATPase and translocation activities also appear to be directly affected by pyrimidine-specific sequence preferences independent of primary site interactions (36-38).

The differences in secondary-site RNA binding seen here between poly-U and poly-A bound forms of Rho (along with the preferential ring closure capability of poly-U over poly-A described in the accompanying study (47) indicate that sequence discrimination in the helicase may in part arise from interrogation of RNA sequence at the point where the nucleic acid threads into the ring. In particular, the $3^{\prime}$ kink seen in the new Rho ${ }^{\mathrm{PolyU}-\mathrm{P} 2_{1}}$ structure allows a ribose $2^{\prime}$ $\mathrm{OH}$ and two pyrimidine bases to interact with an invariant lysine,

allows for the modeling of eight bases of RNA (orange/white). Compared with the Rho ${ }^{\text {PolyU-P }}{ }_{1}$ structure, there is additional density for two $3^{\prime}$ bases in a kinked conformation. (C) The Rho ${ }^{\text {PolyU-P2 }_{1}}$ structure allows for modeling of seven bases of RNA (orange/white). Compared with the Rho ${ }^{\text {Empty }}$ structure, density is lacking for the $5^{\prime}$ base. This structure reveals a hydrogen-bonding network stabilizing interactions between $\mathrm{R}$-loop residue $\mathrm{K} 326$ of subunits $\mathrm{B}$ and $\mathrm{C}$, a water molecule, and the $2^{\prime}-\mathrm{OH}$ and uracil base of the bound RNA kink. $(D)$ The structure of Rho PolyA solved under similar conditions to Rho ${ }^{\text {PolyU-P2 }_{1}}$ also reveals two additional $3^{\prime}$ bases, but in a pseudo A-form helical arrangement, rather than the kinked conformation. In contrast to the $\mathrm{H}$-bonding network observed with the kinked PolyU substrate, no additional protein-RNA contacts are formed.

Fig. 5. Comparison of RNA binding among closed-ring Rho structures. The five core RNA bases visible in all structures are colored white, and additional $5^{\prime}$ and $3^{\prime}$ bases are colored orange. Arrows at the $5^{\prime}$ and $3^{\prime}$ end of the bound RNA indicate the direction of RNA translocation relative to the protein. Protein subunits are colored as in Fig. 2A. Insets reveal proteinRNA hydrogen-bonding details (dashed lines) at the $B / C$ subunit interface. (A) Rho ${ }^{\text {PolyU-P1 }}$ contains six bases of RNA (orange/white) bound by the Rho $\mathrm{Q}$ and $\mathrm{R}$ loops in a spiral staircase conformation. $(B)$ The Rho ${ }^{\text {Empty }}$ structure

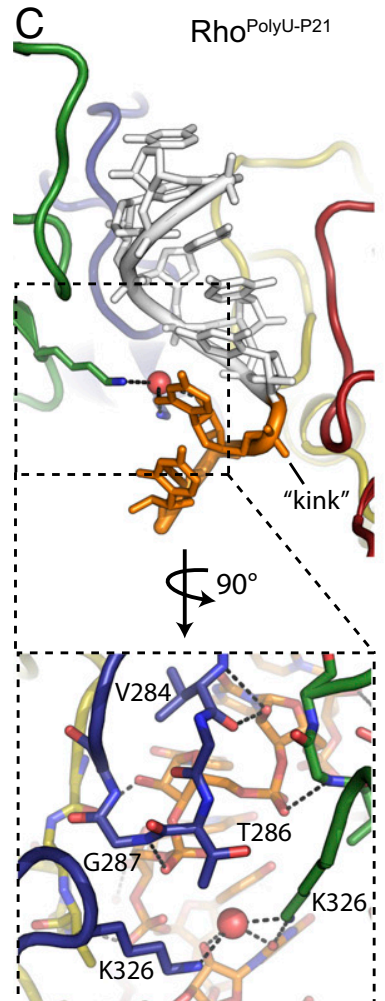


A
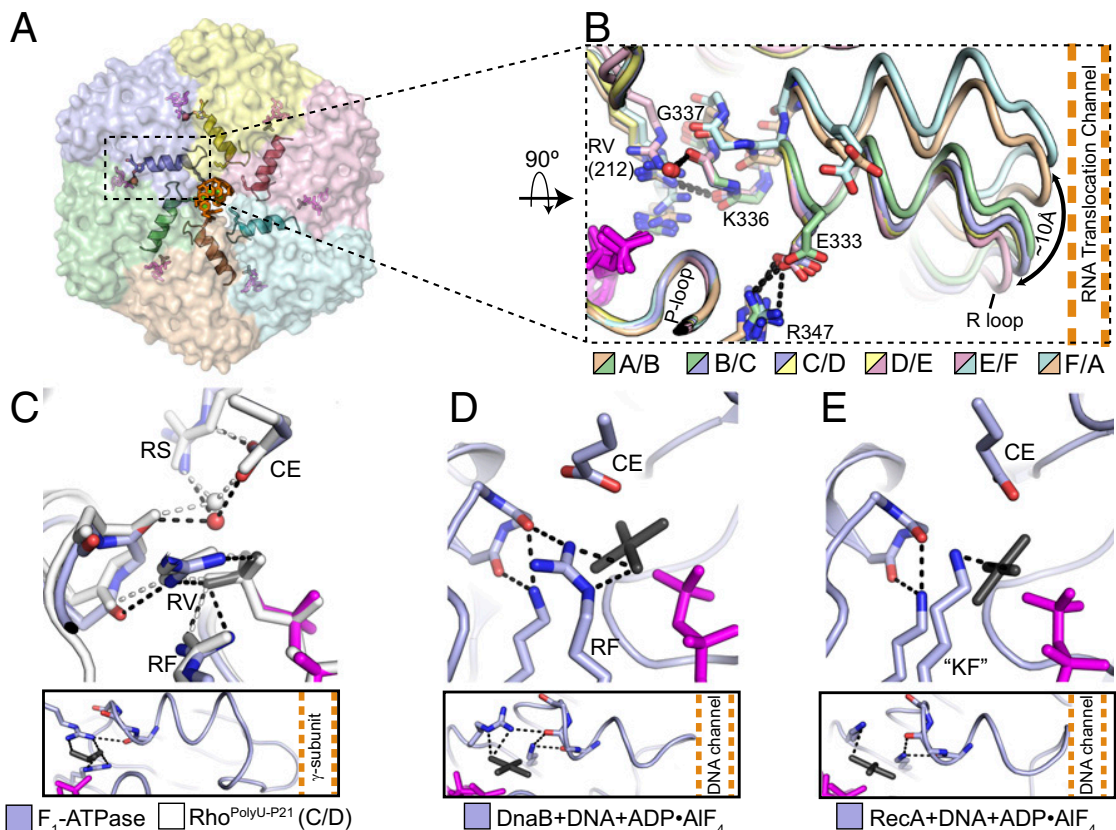

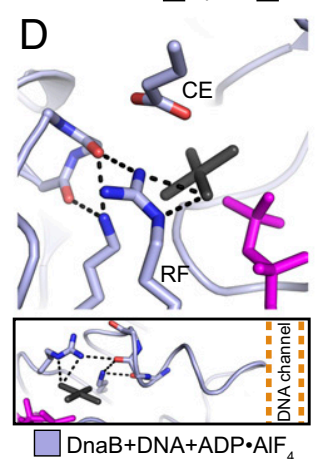

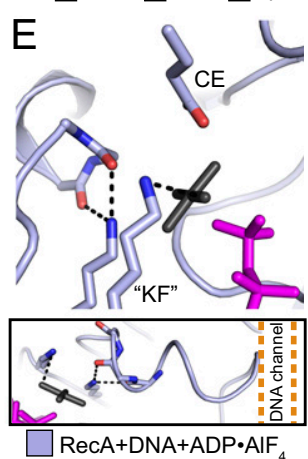

Fig. 6. A conserved allosteric helix couples pore loop movement and ATPase status in RecA-like hexameric motors. (A) An $\alpha$-helix (R 1 ; cartoon) in each subunit of the Rho hexameric motor domain (transparent surface) directly connects the RNAand ATP-binding sites. RNA, orange; nucleotide, magenta; catalytic water molecules, red spheres (subunits B and C only). Lys-326 (colored sticks) directly connects the allosteric helix to bound RNA (B) Alignment of all active sites in Rho ${ }^{\text {PolyU-P2 }_{1}}$ reveals the consequences of intersubunit conformational transitions in the allosteric helix. The helix itself directly links the RNA-binding $\mathrm{R}$ loops in the center of the ring to the position of the $\gamma$-phosphate-sensing residues in the ATP-binding sites at the periphery of the ring, as well as to the catalytic water in the $T^{*}$ active sites. Dashed lines (colored by interface) show bonding interactions present in the $B / C, C / D$, and $D / E$ subunit interfaces. (C) Alignment of the catalytic site in the C/D subunit interface of Rho Polyu-P2 1 (white) with the catalytic site in the structure of the $F_{1}$ ATPase bound to ADP. BeF 3 (colored; PDB ID code 1WOJ) reveals nearly identical alignments of the catalytic residues and the backbone carbonyl groups of the allosteric helix (62). (C, Lower) Position of the $F_{1}$ allosteric helix with respect to the rotating $\gamma$-subunit shaft. (D) The structure of DnaB bound to nucleic acid and GDP.AlF ${ }_{4}$ reveals interactions between its analog of helix $\alpha \alpha 1$ and $\gamma$-phosphate-sensing residues (31). (E) The structure of RecA bound to

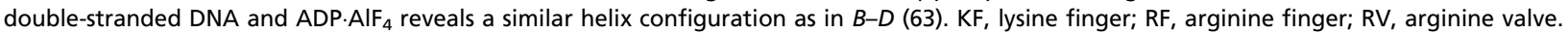

Lys-326, on two different $\mathrm{R}$ loops. These contacts are absent in the $3^{\prime}$ RNA region when Rho is bound to poly-A and in the original Rho ${ }^{\text {PolyU-P1 }}$ model (where the $3^{\prime}$ nucleotides are unstructured; Fig. 5). Intriguingly, the lysines involved in binding the U-U kink in Rho ${ }^{\text {PolyU-P2 }_{1}}$ project from subunits $\mathrm{B}$ and $\mathrm{C}$, which also manifest the two $T^{*}$ states observed in the hexamer (Fig. $2 A$ ). These interactions suggest that Lys-326, in addition to its role neutralizing the backbone charge of RNA bound in Rho's translocation pore (29), may also serve as a sensor for RNA sequence to help properly organize the B- and C-subunit active sites (Fig. $3 A$ ). Such a function would explain why pyrimidine-rich rut sites are particularly well-suited as secondary site ligands to promote nucleotide hydrolysis by Rho, in that they stabilize an RNA structural motif that reciprocally helps set up an idealized ATPase configuration.

The differences in the $3^{\prime}$ RNA structure seen here for the purine and pyrimidine substrates likewise have implications for understanding how RNA sequence is coupled to stepping efficiency. For instance, by using nucleotide analog interference mapping (NAIM) to insert dNTPs into RNA, Rho has been found to be inhibited by the presence of deoxyribose at intervals of every seven nucleotides, with the level of inhibition dependent on the local sequence environment (36). Our structures reveal that pyrimidine, but not purine substrates, can specifically interact with Lys-236 of Rho by forming a specific kink that involves the $2^{\prime}-\mathrm{OH}$ and pyrimidine base of the incoming $3^{\prime}$-RNA end (Fig. 5). Given that movements of the R-loop are directly coupled to changes in the allosteric network that couples ATPase status with RNA binding (Fig. 6 and SI Appendix, Fig. S6) (29), the ability to adopt a kink by pyrimidines may help underpin the positional and sequence dependency of the $2^{\prime}-\mathrm{OH}$ activation step seen to occur approximately every seven steps by NAIM $(36,70)$.

Structural Snapshots Reveal Translocation Substeps in a Hexameric Motor Protein. The present study provides the most comprehensive structural analysis to date for a hexameric helicase bound to different nucleic acid substrates and nucleotide intermediates. In comparing the original Rho ${ }^{\text {PolyU-P1 }}$ and the new Rho ${ }^{\mathrm{PolyU}_{-} \mathrm{P}_{1}}$ models of Rho, both hexamers can be seen to possess a single $E$-state active site (subunit E) that sits adjacent to a $T$ (ATP-bound) state ATPase center (subunit D). The two structures diverge, however, in that the Rho ${ }^{\text {PolyU-P1 }}$ form contains a second $T$-state active site (subunit $\mathrm{C}$ ) followed by two hydrolysis-like $T^{*}$ states (subunits $\mathrm{B}$ and $\mathrm{A}$ ), whereas the Rho ${ }^{\mathrm{PolyU}-\mathrm{P} 2}{ }_{1}$ model is shifted, possessing a lone $T$-state followed immediately by two $T^{*}$ catalytic centers (Fig. 2). As a consequence, the Rho ${ }^{\text {PolyU-P1 }}$ structure contains only a single product $(D)$ state (subunit $\mathrm{F}$ ), whereas the corresponding Rho ${ }^{\mathrm{PolyU}_{-} \mathrm{P}_{1}}$ structure contains two (subunits $\mathrm{F}$ and A). This observation is consistent with prior studies showing that there exists a 2- to 4-subunit delay in ATP hydrolysis and product release in Rho and related RecA-family hexameric helicases such as T7 gp4 (ref. 71 and SI Appendix, SI Discussion).

Although the $E$-state active site (subunit E) remains the most solvent-accessible ATPase center in both crystal forms of Rho, changes in the relative disposition of key functional amino acids are nonetheless evident for this region between the Rho ${ }^{\text {PolyU-P1 }}$ and Rho ${ }^{\mathrm{PolyU}_{-} \mathrm{P}_{1}}$ structures. In particular, the subunit $\mathrm{E}$ active site of the new Rho ${ }^{\text {PolyU-P2 }}{ }_{1}$ model exhibits a displaced and offline catalytic glutamate, but forms a new contact between the arginine finger and the $\mathrm{BeF}_{3}$ moiety; by contrast, in the original Rho ${ }^{\text {PolyU-P1 }}$ structure, the correct positioning of the catalytic glutamate is maintained but the arginine-finger/nucleotide contact is broken (Fig. $3 D$ and $E$ ). Concomitant with these changes, two bases at the $3^{\prime}$ end of the bound RNA become ordered and coordinated in the Rho ${ }^{\text {PolyU-P2 }}{ }_{1}$ structure, whereas in the Rho ${ }^{\text {PolyU-P1 }}$ model, only a single extra base is apparent at the $5^{\prime}$ RNA end (Fig. 5). Interestingly, back-soaking of the new poly-U Rho crystal form, which results in nucleotide dissociation from the E-subunit interface, produces a hybrid state in which RNA density is evident at both the $5^{\prime}$ and $3^{\prime}$ ends.

Together, the suite of Rho structures not only recapitulates the movements expected for $5^{\prime} \rightarrow 3^{\prime}$ translocation along RNA, but also highlights the existence of substeps within the context of a sequential ATPase mechanism (Fig. 7 and Movie S3). In this scheme, formation of an empty ATPase center would promote downstream $\left(3^{\prime}\right)$ contacts between Rho and its substrate, while maintaining $5^{\prime}$ interactions leftover from the prior round of hydrolysis. ATP binding would next disrupt the 5' RNA contact, initiating a new translocation cycle to pull the $3^{\prime}$ end of the 


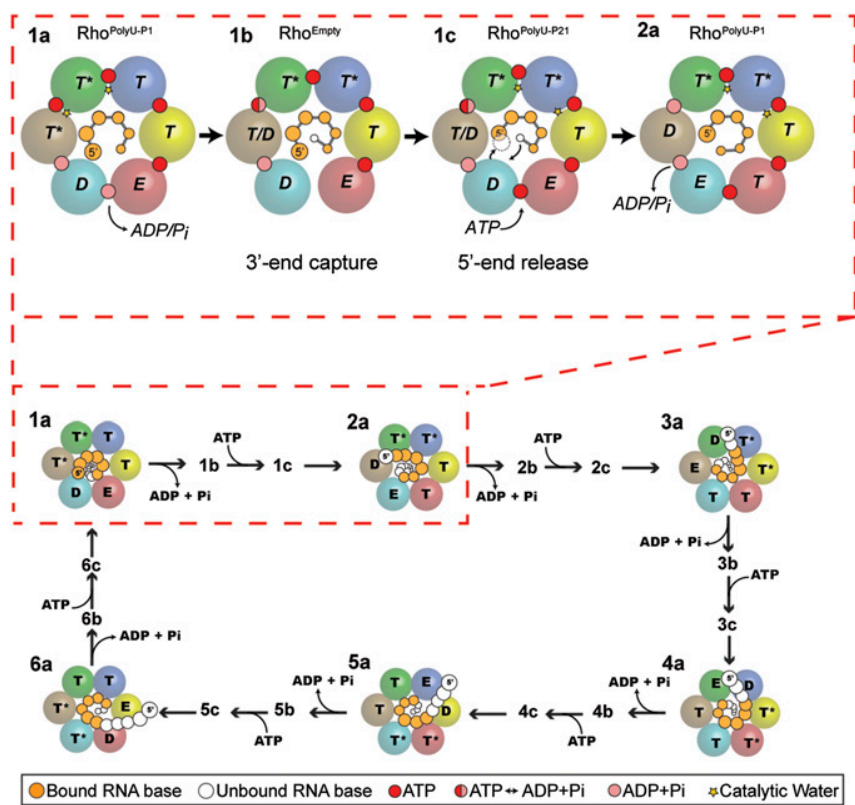

Fig. 7. Substepping in a hexameric helicase. (Upper, red dashed box) Diagram of RNA bases and catalytic states in the Rho ${ }^{\text {PolyU-P1 }}$, Rho ${ }^{\text {PolyU-P2 }_{1} \text {, and }}$ $\mathrm{Rho}^{\text {Empty }}$ structures. Protein subunits are shown as large spheres colored by chain as in Fig. 2A. Arrows indicate the sequence of proposed substeps in which an incoming $3^{\prime}$ RNA base is bound and a $5^{\prime}$ RNA base is released, concomitant with the release of nucleotide and a shift in position of the $T^{\star}$ catalytic states. Yellow stars designate the sites of the nucleophilic water observed in the various Rho structures; only in the Rho ${ }^{\text {PolyU-P2 }_{1}}$ structure does this water adopt an in-line attack position. (Lower) Relationship of the substeps to a complete series of six sequential hydrolysis and translocation steps by Rho.

bound RNA into the motor pore. Finally, product formation would complete the translocation step, helping to shepherd the $5^{\prime}$ RNA end out of the helicase, after which ADP release would reset the system by forming new contacts to the incoming $3^{\prime}$ base.

Although conformational substeps have been observed structurally for superfamily 1 and 2 helicases and for motors such as the F1 ATPase, myosin, and kinesin (72-75), similar insights have not been forthcoming for hexameric helicase/translocase systems. Functional substeps (as well as macrosteps) are frequently observed in single-molecule studies of motor proteins $(76,77)$, but the architectural underpinnings of these events often remained unresolved. The present work demonstrates the existence of distinct physical substates in a RecA-family hexameric helicase and, moreover, implicates both RNA binding and different stages of the ATPase cycle in the formation and dissolution of these states. Future efforts involving high-resolution single-molecule studies and structure-guided mutagenesis are needed to establish the timing by which different states interconvert with each other and the role that specific, protein-

1. Delagoutte E, von Hippel PH (2003) Helicase mechanisms and the coupling of helicases within macromolecular machines. Part II: Integration of helicases into cellular processes. Q Rev Biophys 36(1):1-69.

2. Patel SS, Picha KM (2000) Structure and function of hexameric helicases. Annu Rev Biochem 69:651-697.

3. Singleton MR, Dillingham MS, Wigley DB (2007) Structure and mechanism of helicases and nucleic acid translocases. Annu Rev Biochem 76:23-50.

4. Sauer RT, Baker TA (2011) AAA+ proteases: ATP-fueled machines of protein destruction. Annu Rev Biochem 80:587-612.

5. Ahnert P, Picha KM, Patel SS (2000) A ring-opening mechanism for DNA binding in the central channel of the T7 helicase-primase protein. EMBO J 19(13): 3418-3427.

6. O'Shea VL, Berger JM (2014) Loading strategies of ring-shaped nucleic acid translocases and helicases. Curr Opin Struct Biol 25:16-24. mediated contacts between RNA and ATP play in controlling these transitions.

\section{Methods}

SAXS Data Collection and Analysis. Native Rho protein was prepared in a similar manner to selenomethionine-labeled protein as described (22). Rho protein $(40 \mathrm{mg} / \mathrm{mL})$ in size-exclusion buffer [50 mM Tris pH 7.5, $500 \mathrm{mM} \mathrm{KCl}$, $10 \%$ (vol/vol) glycerol, and $1 \mathrm{mM}$ Tris(2-carboxyethyl)phosphine (TCEP)) was dialyzed overnight into SAXS buffer $(150 \mathrm{mM} \mathrm{KCl}, 50 \mathrm{mM}$ Hepes $\mathrm{pH}$ 7.5, $5 \mathrm{mM}$ $\mathrm{MgCl}_{2}$, and $5 \%$ (vol/vol) glycerol). Dialyzed protein was then further diluted to a concentration of $5 \mathrm{mg} / \mathrm{mL}$ in SAXS buffer containing various concentrations of RNA (rU12) or ADP.BeF 3 , aliquoted into a 96-well plate, and used for data collection at room temperature using an automated system at Advanced Light Source Beamline 12.3.1. Raw SAXS data were integrated, scaled, and buffersubtracted with the program OGRE (Greg Hura, Lawrence Berkeley National Laboratory, Berkeley, CA). PRIMUS was used to merge data from a 1- and 10-s exposure and to manually remove outliers near the beam stop to obtain highquality scattering curves with very little noise (43). Guinier analysis was performed by using AUTORG (78). Distance distribution functions $[P(r)]$, maximum diameters $\left(D_{\text {max }}\right)$, and real- and reciprocal-space radius of gyration $\left(R_{\mathrm{g}}\right)$ values were calculated with GNOM (79). Structural models were generated by using an open-ring Rho model [PDB ID code 1PVO (22)] and the closed-ring crystal structure determined here (Rho ${ }^{\text {PolyU-P2 }}{ }_{1}$ ). Missing loops or domains were added to the structural models by superposition with other structures or subunits containing the missing residues. OLIGOMER and FOXS were used to compare theoretical scattering curves to the data and compute the relative contributions of multicomponent datasets $(44,45,80)$. Background subtraction as implemented in FOXS or OLIGOMER was used to account for errors in buffer composition and improve the fit of the high-resolution data.

Protein Crystallization and Data Collection. Selenomethionine-labeled E. coli Rho protein was prepared, and the RNA-ADP.BeF ${ }_{3}$ complexes were formed as described $(22,29)$, except that Rho was dialyzed into a buffer containing $10 \mathrm{mM}$ Tris (pH 7.5), $100 \mathrm{mM} \mathrm{NaCl}$, and $0.5 \mathrm{mM}$ TCEP before complex formation. RNA (rU12 and rA12 polymers) was purchased from IDT. See SI Appendix, SI Methods for a detailed description of crystallization, harvesting, and data collection.

Structure Solution and Refinement. A detailed description of data processing, phase determination, and model refinement used to solve Rho ${ }^{\text {PolyU-P2 }}$, Rho ${ }^{\text {Empty }}$, and Rho ${ }^{\text {PolyA }}$ structures can be found in SI Appendix, SI Methods.

Structural Analysis. All figures were prepared by using PyMOL (Schrödinger). Structural superpositions were conducted with the CEALIGN PyMOL plugin (81). Energy-minimized linear interpolations were computed by using a CNS script written by the Yale Morph Server (82). To place atomic B factors from different structures on the same relative scale (SI Appendix, Fig. S4), atomic B factors for each structure were divided by the average $B$ factor for that structure and then multiplied by the average B factor for all structures. Distance vectors between various Rho structures were calculated as described (83). Vector length corresponds to distances between $\mathrm{C} \alpha$ atoms among different structures multiplied by a scale factor of three to assist visualization, and vector color corresponds to relative distance (dark blue, $0 \AA$; red, $1.5 \AA$ or greater).

ACKNOWLEDGMENTS. We thank members of the J.M.B. laboratory for helpful discussions; Greg Hura and Dina Schneidman-Duhovny for guidance on SAXS data processing; Jamie Cate for assistance with distance vector calculations; and Jane Tanamachi, George Meigs, and James Holton at ALS Beamline 8.3.1 and Michal Hammel at ALS Beamline 12.3.1 for assistance with SAXS data collection. This research was supported by the G. Harold and Leila Y. Mathers Foundation and NIH Grant GM071747 (to J.M.B.).

7. Davey MJ, Jeruzalmi D, Kuriyan J, O'Donnell M (2002) Motors and switches: AAA+ machines within the replisome. Nat Rev Mol Cell Bio/ 3(11):826-835.

8. Richardson JP (2002) Rho-dependent termination and ATPases in transcript termination. Biochim Biophys Acta 1577(2):251-260.

9. Boudvillain M, Nollmann M, Margeat E (2010) Keeping up to speed with the transcription termination factor Rho motor. Transcription 1(2):70-75.

10. Dombroski AJ, Platt T (1988) Structure of rho factor: An RNA-binding domain and a separate region with strong similarity to proven ATP-binding domains. Proc Natl Acad Sci USA 85(8):2538-2542.

11. Bear DG, et al. (1985) Escherichia coli transcription termination factor rho has a two-domain structure in its activated form. Proc Natl Acad Sci USA 82(7): 1911-1915.

12. Chen CY, Richardson JP (1987) Sequence elements essential for rho-dependent transcription termination at lambda tR1. J Biol Chem 262(23):11292-11299. 
13. Peters JM, et al. (2012) Rho and NusG suppress pervasive antisense transcription in Escherichia coli. Genes Dev 26(23):2621-2633.

14. Epshtein V, Dutta D, Wade J, Nudler E (2010) An allosteric mechanism of Rho-dependent transcription termination. Nature 463(7278):245-249.

15. Brennan CA, Dombroski AJ, Platt T (1987) Transcription termination factor rho is an RNA-DNA helicase. Cell 48(6):945-952.

16. Park JS, Roberts JW (2006) Role of DNA bubble rewinding in enzymatic transcription termination. Proc Natl Acad Sci USA 103(13):4870-4875.

17. Lau LF, Roberts JW, Wu R (1983) RNA polymerase pausing and transcript release at the lambda tR1 terminator in vitro. J Biol Chem 258(15):9391-9397.

18. Morgan WD, Bear DG, von Hippel PH (1983) Rho-dependent termination of transcription. II. Kinetics of mRNA elongation during transcription from the bacteriophage lambda PR promoter. J Biol Chem 258(15):9565-9574.

19. Koslover DJ, Fazal FM, Mooney RA, Landick R, Block SM (2012) Binding and translocation of termination factor rho studied at the single-molecule level. J Mol Biol 423(5):664-676.

20. Gogol EP, Seifried SE, von Hippel PH (1991) Structure and assembly of the Escherichia coli transcription termination factor rho and its interaction with RNA. I. Cryoelectron microscopic studies. J Mol Biol 221(4):1127-1138.

21. Bear DG, et al. (1988) Interactions of Escherichia coli transcription termination factor rho with RNA. II. Electron microscopy and nuclease protection experiments. J Mol Biol 199(4):623-635.

22. Skordalakes E, Berger JM (2003) Structure of the Rho transcription terminator: Mechanism of mRNA recognition and helicase loading. Cell 114(1):135-146.

23. Skordalakes E, Berger JM (2006) Structural insights into RNA-dependent ring closure and ATPase activation by the Rho termination factor. Cell 127(3):553-564.

24. Yu X, Horiguchi T, Shigesada K, Egelman EH (2000) Three-dimensional reconstruction of transcription termination factor rho: Orientation of the $\mathrm{N}$-terminal domain and visualization of an RNA-binding site. J Mol Biol 299(5):1279-1287.

25. Richardson JP (2003) Loading Rho to terminate transcription. Cell 114(2):157-159.

26. Costa A, et al. (2011) The structural basis for MCM2-7 helicase activation by GINS and Cdc45. Nat Struct Mol Biol 18(4):471-477.

27. Lyubimov AY, Costa A, Bleichert F, Botchan MR, Berger JM (2012) ATP-dependent conformational dynamics underlie the functional asymmetry of the replicative helicase from a minimalist eukaryote. Proc Natl Acad Sci USA 109(30):11999-12004.

28. Bochman ML, Schwacha A (2010) The Saccharomyces cerevisiae $\mathrm{Mcm} 6 / 2$ and $\mathrm{Mcm} 5 / 3$ ATPase active sites contribute to the function of the putative Mcm2-7 'gate'. Nucleic Acids Res 38(18):6078-6088.

29. Thomsen ND, Berger JM (2009) Running in reverse: The structural basis for trans location polarity in hexameric helicases. Cell 139(3):523-534.

30. Enemark EJ, Joshua-Tor L (2006) Mechanism of DNA translocation in a replicative hexameric helicase. Nature 442(7100):270-275.

31. Itsathitphaisarn O, Wing RA, Eliason WK, Wang J, Steitz TA (2012) The hexameric helicase DnaB adopts a nonplanar conformation during translocation. Cell 151(2):267-277.

32. Singleton MR, Sawaya MR, Ellenberger T, Wigley DB (2000) Crystal structure of T7 gene 4 ring helicase indicates a mechanism for sequential hydrolysis of nucleotides. Cell 101(6):589-600.

33. Abrahams JP, Leslie AG, Lutter R, Walker JE (1994) Structure at 2.8 A resolution of F1ATPase from bovine heart mitochondria. Nature 370(6491):621-628.

34. Costa A, et al. (2014) DNA binding polarity, dimerization, and ATPase ring remodeling in the CMG helicase of the eukaryotic replisome. eLife 3:e03273.

35. Rothenberg E, Trakselis MA, Bell SD, Ha T (2007) MCM forked substrate specificity involves dynamic interaction with the 5'-tail. J Biol Chem 282(47):34229-34234.

36. Schwartz A, et al. (2009) A stepwise 2'-hydroxyl activation mechanism for the bacterial transcription termination factor Rho helicase. Nat Struct Mol Biol 16(12):1309-1316.

37. Wang Y, von Hippel PH (1993) Escherichia coli transcription termination factor rho. I. ATPase activation by oligonucleotide cofactors. J Biol Chem 268(19):13940-13946.

38. Richardson JP (1982) Activation of rho protein ATPase requires simultaneous interaction at two kinds of nucleic acid-binding sites. J Biol Chem 257(10):5760-5766.

39. Soares E, Schwartz A, Nollmann M, Margeat E, Boudvillain M (2014) The RNA-mediated, asymmetric ring regulatory mechanism of the transcription termination Rho helicase decrypted by time-resolved nucleotide analog interference probing (trNAIP). Nucleic Acids Res 42(14):9270-9284.

40. Putnam CD, Hammel M, Hura GL, Tainer JA (2007) X-ray solution scattering (SAXS) combined with crystallography and computation: Defining accurate macromolecular structures, conformations and assemblies in solution. Q Rev Biophys 40(3):191-285.

41. Rambo RP, Tainer JA (2013) Accurate assessment of mass, models and resolution by small-angle scattering. Nature 496(7446):477-481.

42. Capp JA, Hagarman A, Richardson DC, Oas TG (2014) The statistical conformation of a highly flexible protein: Small-angle x-ray scattering of $S$. aureus protein A. Structure 22(8):1184-1195.

43. Konarev PV, Volkov VV, Sokolova AV, Koch MHJ, Svergun DI (2003) PRIMUS: A Windows PC-based system for small-angle scattering data analysis. J Appl Cryst 36:1277-1282.

44. Schneidman-Duhovny D, Hammel M, Tainer JA, Sali A (2013) Accurate SAXS profile computation and its assessment by contrast variation experiments. Biophys $J$ 105(4):962-974.

45. Schneidman-Duhovny D, Hammel M, Sali A (2010) FoXS: A web server for rapid computation and fitting of SAXS profiles. Nucleic Acids Res 38(Web Server issue):W540-544.

46. Jeong YJ, Kim DE, Patel SS (2004) Nucleotide binding induces conformational changes in Escherichia coli transcription termination factor Rho. J Biol Chem 279(18) 18370-18376.

47. Lawson MR, Dyer K, Berger JM (2016) Ligand-induced and small molecule control of substrate loading in a hexameric helicase. Proc Natl Acad Sci USA 113:13715-13720.

48. Thomsen ND, Berger JM (2012) Crystallization and x-ray structure determination of an RNA-dependent hexameric helicase. Methods Enzymol 511:171-190.
49. Frauenfelder H, Petsko GA, Tsernoglou D (1979) Temperature-dependent x-ray diffraction as a probe of protein structural dynamics. Nature 280(5723):558-563.

50. Geiselmann J, von Hippel PH (1992) Functional interactions of ligand cofactors with Escherichia coli transcription termination factor rho. I. Binding of ATP. Protein Sci 1(7): 850-860.

51. Kim DE, Patel SS (1999) The mechanism of ATP hydrolysis at the noncatalytic sites of the transcription termination factor Rho. J Biol Chem 274(46):32667-32671.

52. Kim DE, Shigesada K, Patel SS (1999) Transcription termination factor Rho contains three noncatalytic nucleotide binding sites. J Biol Chem 274(17):11623-11628.

53. Seifried SE, Easton JB, von Hippel PH (1992) ATPase activity of transcriptiontermination factor rho: Functional dimer model. Proc Natl Acad Sci USA 89(21) 10454-10458.

54. Stitt BL (1988) Escherichia coli transcription termination protein rho has three hydrolytic sites for ATP. J Biol Chem 263(23):11130-11137

55. Xu Y, Johnson J, Kohn H, Widger WR (2003) ATP binding to Rho transcription termination factor. Mutant F355W ATP-induced fluorescence quenching reveals dynamic ATP binding. J Biol Chem 278(16):13719-13727.

56. Rabhi M, et al. (2011) Mutagenesis-based evidence for an asymmetric configuration of the ring-shaped transcription termination factor Rho. J Mol Biol 405(2):497-518.

57. Miwa Y, Horiguchi T, Shigesada K (1995) Structural and functional dissections of transcription termination factor rho by random mutagenesis. J Mol Biol 254(5): 815-837.

58. Wei RR, Richardson JP (2001) Identification of an RNA-binding site in the ATP binding domain of Escherichia coli Rho by H2O2/Fe-EDTA cleavage protection studies. J Biol Chem 276(30):28380-28387.

59. $\mathrm{Xu} \mathrm{Y,} \mathrm{Kohn} \mathrm{H,} \mathrm{Widger} \mathrm{WR} \mathrm{(2002)} \mathrm{Mutations} \mathrm{in} \mathrm{the} \mathrm{rho} \mathrm{transcription} \mathrm{termination}$ factor that affect RNA tracking. J Biol Chem 277(33):30023-30030.

60. Balasubramanian K, Stitt BL (2010) Evidence for amino acid roles in the chemistry of ATP hydrolysis in Escherichia coli Rho. J Mol Biol 404(4):587-599.

61. Lowery-Goldhammer C, Richardson JP (1974) An RNA-dependent nucleoside triphosphate phosphohydrolase (ATPase) associated with rho termination factor. Proc Natl Acad Sci USA 71(5):2003-2007.

62. Kagawa R, Montgomery MG, Braig K, Leslie AG, Walker JE (2004) The structure of bovine F1-ATPase inhibited by ADP and beryllium fluoride. EMBO J 23(14):2734-2744.

63. Chen Z, Yang H, Pavletich NP (2008) Mechanism of homologous recombination from the RecA-ssDNA/dsDNA structures. Nature 453(7194):489-494.

64. Lowry $\mathrm{OH}$, Carter J, Ward JB, Glaser L (1971) The effect of carbon and nitrogen sources on the level of metabolic intermediates in Escherichia coli. J Biol Chem 246(21):6511-6521.

65. Bennett BD, et al. (2009) Absolute metabolite concentrations and implied enzyme active site occupancy in Escherichia coli. Nat Chem Biol 5(8):593-599.

66. Albe KR, Butler MH, Wright BE (1990) Cellular concentrations of enzymes and their substrates. J Theor Biol 143(2):163-195.

67. Bailey S, Eliason WK, Steitz TA (2007) Structure of hexameric DnaB helicase and its complex with a domain of DnaG primase. Science 318(5849):459-463.

68. Sanders CM, et al. (2007) Papillomavirus E1 helicase assembly maintains an asymmetric state in the absence of DNA and nucleotide cofactors. Nucleic Acids Res 35(19): 6451-6457.

69. Johnson DS, Bai L, Smith BY, Patel SS, Wang MD (2007) Single-molecule studies reveal dynamics of DNA unwinding by the ring-shaped T7 helicase. Cell 129(7):1299-1309.

70. Patel SS (2009) Structural biology: Steps in the right direction. Nature 462(7273): 581-583.

71. Liao J-C, Jeong Y-J, Kim D-E, Patel SS, Oster G (2005) Mechanochemistry of t7 DNA helicase. J Mol Biol 350(3):452-475.

72. Kull FJ, Sablin EP, Lau R, Fletterick RJ, Vale RD (1996) Crystal structure of the kinesin motor domain reveals a structural similarity to myosin. Nature 380(6574):550-555.

73. Sablin EP, et al. (1998) Direction determination in the minus-end-directed kinesin motor ncd. Nature 395(6704):813-816.

74. Schmidt H, Zalyte R, Urnavicius L, Carter AP (2015) Structure of human cytoplasmic dynein-2 primed for its power stroke. Nature 518(7539):435-438.

75. Rees DM, Montgomery MG, Leslie AG, Walker JE (2012) Structural evidence of a new catalytic intermediate in the pathway of ATP hydrolysis by F1-ATPase from bovine heart mitochondria. Proc Natl Acad Sci USA 109(28):11139-11143.

76. Chistol G, et al. (2012) High degree of coordination and division of labor among subunits in a homomeric ring ATPase. Cell 151(5):1017-1028.

77. Yasuda R, Noji H, Yoshida M, Kinosita K, Jr, Itoh H (2001) Resolution of distinct rotational substeps by submillisecond kinetic analysis of F1-ATPase. Nature 410(6831) 898-904.

78. Petoukhov MV, Konarev PV, Kikhney AG, Svergun DI (2007) ATSAS 2.1-towards automated and web-supported small-angle scattering data analysis. J Appl Cryst 40: s223-s228.

79. Svergun DI (1992) Determination of the regularization parameter in indirect-transform methods using perceptual criteria. J App/ Cryst 25:495-503.

80. Svergun D, Barberato C, Koch MHJ (1995) CRYSOL-a program to evaluate x-ray solution scattering of biological macromolecules from atomic coordinates. J Appl Cryst 28(6):768-773.

81. Shindyalov IN, Bourne PE (1998) Protein structure alignment by incremental combinatorial extension (CE) of the optimal path. Protein Eng 11(9):739-747.

82. Flores S, et al. (2006) The Database of Macromolecular Motions: New features added at the decade mark. Nucleic Acids Res 34(Database issue):D296-D301.

83. Zhang W, Dunkle JA, Cate JH (2009) Structures of the ribosome in intermediate states of ratcheting. Science 325(5943):1014-1017. 